\title{
New Universality of the Metal-Insulator Transition in an Integer Quantum Hall Effect System
}

\author{
D. N. Sheng and Z. Y. Weng \\ Texas Center for Superconductivity, University of Houston, Houston, Texas 77204-5506
}

(Received 8 September 1997)

\begin{abstract}
A new universality of the metal-insulator transition in an integer quantum Hall effect (IQHE) system is studied, based on a lattice model, where the IQHE states exist only within a finite range of Fermi energy in the presence of disorders. A two-parameter scaling law is found at the high-energy boundary where direct transitions from high filling-factor IQHE states to an insulator occur. We find that $\rho_{x x}=\rho_{x y}$ at the critical point whose value can continuously vary as a single function of the Landau-level filling number $n_{\nu}$. Such a new universality well explains the recent experiment by Song et al. [Phys. Rev. Lett. 78, 2200 (1997)]. [S0031-9007(97)05021-7]
\end{abstract}

PACS numbers: 73.40.Hm, 71.30. $+\mathrm{h}, 73.20 . \mathrm{Jc}$

The global phase diagram proposed by Kivelson, Lee, and Zhang [1] (KLZ) for the quantum Hall effect (QHE) system has stimulated a series of recent experiments. Although many of these experiments are in support of the overall picture of KLZ, controversies arise concerning the select rule for metal-insulator transitions in such a system [2-4]. The global phase diagram predicts that the transition from an IQHE state to insulator can occur only at a low-filling number corresponding to the $\nu=1$ integer quantum Hall effect (IQHE) state to insulator $(1 \rightarrow 0)$ transition, while at higher-filling number the only allowed transitions are the nearest-neighbor plateau-plateau transitions $(\nu \rightarrow \nu \pm 1)$. Experimentally, however, direct transitions from $\nu=1, \nu=2, \nu=3$, and $\nu=6$ IQHE states to insulator have all been observed recently [2-4] on the highfilling number side. Although the apparent inconsistency of the $\nu=2$ IQHE state to insulator transition with the global phase diagram may be reconciled by assuming that the lowest Landau level (LL) is spin degenerate [5], the higher plateaus to insulator transitions $(3 \rightarrow 0$ and $6 \rightarrow 0)$ cannot be explained similarly due to the fact that these transitions must take place at higher LL's.

The select rule of the global phase diagram is based on the so-called float-up picture [6,7] where extended states in the weak field limit are assumed to shift toward higher energy indefinitely without disruption. But recent numerical calculations $[8,9]$ have shown that the float-up picture is not correct in a lattice model in which extended states merge and disappear before they can reach to the band center. This non-float-up picture holds down to very weak magnetic field limit [9] which means that the lattice model is not equivalent to the continuum model even in the limit where the magnetic length scale is much larger than the lattice constant. This surprising result, which can be well understood based on a topological Chern number description [9], implies that the lattice effect will always remain to be an important factor in the problem of metal-insulator transitions even in the weak field limit. It may thus provide an explanation for the aforementioned experimental observations of a direct transition from the higher IQHE states to an insulator.
Recent experimental measurement has further indicated [4] that the direct transition from a higher IQHE state to insulator may belong to a new universality. In the corresponding critical regime, the longitudinal and transverse conductances generally satisfy $\sigma_{x x c}=\sigma_{x y c}$ within the experimental error and their value varies continuously with the filling number, which is different from the usual plateau-plateau transitions (including the $1 \rightarrow 0$ transition as well) in which $\sigma_{x x c}=0.5 e^{2} / h$ and $\sigma_{x y c}=(\nu+$ $0.5) e^{2} / h$ [10]. So far such a new class of metal-insulator transitions in the IQHE system cannot be understood by any existing theory of the IQHE based on the continuum model. On the other hand, since the direct transitions from higher IQHE states to insulator are present naturally in a lattice model as mentioned above, it would be very desirable to examine the corresponding critical behaviors.

In this Letter, we present a numerical study based on the calculation of longitudinal and transverse conductances and a finite-size scaling analysis near these critical points. Our results show that a direct metal-insulator transition in this regime indeed belongs to a new universality. In particular, the scaling behavior of localization length near each critical point obeys a real two-parameter scaling law, in contrast to the plateau-plateau transitions whose critical behavior can be reduced to a one-parameter scaling law [11]. As the consequence, $\sigma_{x x c}$ and $\sigma_{x y c}$, which are equal to each other, continuously vary as a function of a single parameter-the LL filling factor $n_{\nu}$, and does not depend on magnetic field or disorder strength independently. Since such a critical behavior is independent of the strength of magnetic field, we are able to make a close comparison of our numerical results with experimental measurement [4] and find a consistent explanation.

The lattice Hamiltonian to be studied is a tight-binding model (TBM) given as follows:

$$
H=-\sum_{\langle i j\rangle} e^{i a_{i j}} c_{i}^{+} c_{j}+\text { H.c. }+\sum_{i} w_{i} c_{i}^{+} c_{i},
$$

where the hopping integral is taken as the unit, and $c_{i}^{+}$ is a fermionic creation operator with $\langle i j\rangle$ referring to two nearest neighboring sites. A uniform magnetic flux per 
plaquette is given as $\phi=\sum_{\square} a_{i j}=2 \pi / M$, where the summation runs over four links around a plaquette. $w_{i}$ is a random potential with strength $\left|w_{i}\right| \leq W / 2$, and the white noise limit is considered with no correlations among different sites for $w_{i}$.

In the weak disorder limit, a well-defined IQHE plateau structure is exhibited in the Hall conductance. For our purpose, the disorder strength $W$ is continuously increased to identify a direct transition from the $\nu$ th IQHE state to insulator. For instance, in Fig. 1(a), by fixing the Fermi energy near the center of the $\nu=2$ plateau $\left(E_{f}=-2.75\right)$ at weak disorder, we show how the Hall $\left(\sigma_{x y}\right)$ and longitudinal $\left(\sigma_{x x}\right)$ conductances evolve with disorders (at a flux strength $\phi=2 \pi / 16)$. Here $\sigma_{x y}$ is calculated based on the Kubo formula and $\sigma_{x x}$ by the Landauer formula [12]. At weak disorder $(W \leq 1), \sigma_{x y}$ is well quantized at $\nu e^{2} / h$ with $\nu=2$. With $W$ increasing from 1 to $8, \sigma_{x y}$ continuously reduces to zero without showing a $\nu=1$ plateau, indicating a direct $\nu=2 \rightarrow \nu=0$ transition. Two curves for $\sigma_{x y}$ at different sample sizes $\left(N=16^{2}\right.$ and $N=32^{2}$ ) cross at a critical point which also coincides with the maximum point of the longitudinal conductance $\sigma_{x x}$. The latter is also sample-size independent at the corresponding critical disorder strength $W_{c}=3.5$. But away from $W_{c}, \sigma_{x x}$ monotonically decrease with the

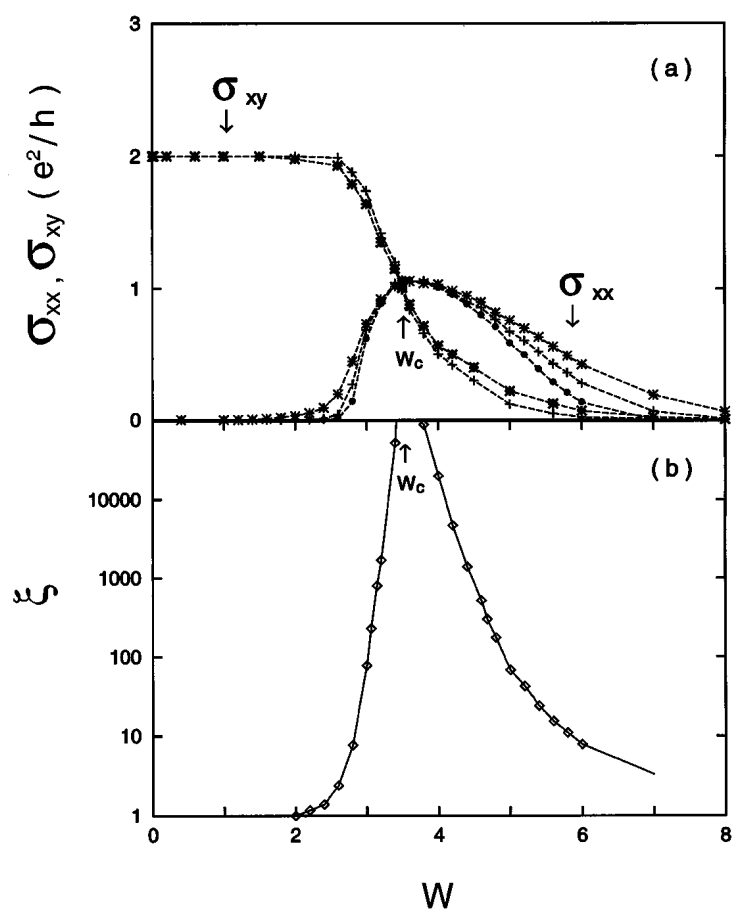

FIG. 1. (a) The evolution of $\sigma_{x x}$ and $\sigma_{x y}$ with disorder strength $W$ at a fixed Fermi energy $E_{f}=-2.75$, which corresponds to the center of the $\nu=2$ IQHE plateau at weak $W$ limit. Lattice sizes vary from $16 \times 16(*), 32 \times 32(+)$, to $64 \times 64(\bullet)$, and the sample-independent fixed point at $W_{c}=3.5$ indicates the crossing of the last extended level at $E_{f}$. (b) Thermodynamic localization length $\xi$ also diverges at $W_{c}$. increase of sample size as a typical behavior in localized states. Thus the existence of a single $W_{c}$ identified from both $\sigma_{x y}$ and $\sigma_{x x}$ confirms that the $\nu=2 \rightarrow 0$ transition is really a one-step transition. Furthermore, a calculation of the finite-size localization length by using the transfer matrix method [13] also verifies the same $W_{c}$ : at the fixed $E_{f}=-2.75$, we find that the localization length satisfying the scaling relation, $\lambda_{L} / L=f^{\prime}(\xi(W) / L)$, for a stripe sample with the width varying from $L=16,24,32$, 48,64 , to 80 . Here $\xi$ is identified as the thermodynamic localization length which diverges at $W_{c}=3.5$ as shown in Fig. 1(b). At such a critical point, we obtain $\sigma_{x x c}=(1.06 \pm 0.02) e^{2} / h$ and $\sigma_{x y c}=(1.01 \pm 0.06) e^{2} / h$, or $\sigma_{x x c}=\sigma_{x y c}$ within the statistics error bar (6\%).

Fixing the Fermi energy $E_{f}$ near the center of the $\nu$ th plateaus at weak $W$, we always find a similar direct transition to insulator $(\nu \rightarrow 0)$ as $W$ increases. The critical disorder $W_{c \nu}$ for $\nu \rightarrow 0$ transition is found to satisfy a sequence $W_{c \nu}<W_{c \nu^{\prime}}$ for $\nu>\nu^{\prime}$. For instance, we have $W_{c 1}=3.8, W_{c 2}=3.5, W_{c 3}=3.2$, and $W_{c 4}=2.8$ at a flux strength $2 \pi / 16$. Up to the weakest flux strength $2 \pi / 384$ that we can get access to, we always find the same sequence for the disappearance of the IQHE plateaus. According to this sequence, near the critical disorder $W_{c \nu}$ for the $\nu$ th plateau, all higher plateaus have already disappeared while lower ones are still robust. We can then define the transition of $\nu \rightarrow 0$ as the boundary of the IQHE regime on the high-energy (or high-filling number) side, beyond which the IQHE states no longer exist up to the band center. The corresponding critical conductances $\sigma_{x x c}$ and $\sigma_{x y c}$ at $W_{c \nu}$ always satisfy the relation $\sigma_{x x c}=$ $\sigma_{x y c}$ within the numerical error bar, and their values continuously vary with the plateau index $\nu$ and the Fermi energy $E_{f}$. Nevertheless, we find that all of them can be scaled into a single curve as the function of the LL filling number $n_{\nu}$ shown in Fig. 2, in which the magnetic flux changes from $2 \pi / 16$ to $2 \pi / 96$. In Fig. 2 , we show $\rho_{x x c}$ and $\rho_{x y c}$ instead of $\sigma_{x x c}$ and $\sigma_{x y c}$ in order to compare with experimental data of Song et al. [4] in the inset in Fig. 2. We see that the overall agreement between the theory and experiment is quite reasonable. (Note that in the inset the top horizontal axis is carrier density which has not been converted to $n_{\nu}$ here as the corresponding magnetic fields are not provided in Ref. [4].) Both $\rho_{x x c}$ and $\rho_{x y c}$ monotolically increase with the decrease of $n_{\nu}$ and are eventually saturated at $h / e^{2}$. We can make a closer comparison: in Ref. [4] the $\nu=3 \rightarrow 0$ transition covers a range of critical resistivities from 0.27 to 0.4 in units of $h / e^{2}$, which agrees well with the theoretical values shown in Fig. 2. The theory also predicts that the transitions reported in the experiment with critical resistivities less than $0.27\left(h / e^{2}\right)$ actually come from higher $(\nu \geq 4)$ IQHE states to insulator transitions.

Thus we have identified a new universality of metalinsulator transition at the boundary between the IQHE regime and insulator on the high-filling-number (energy) 


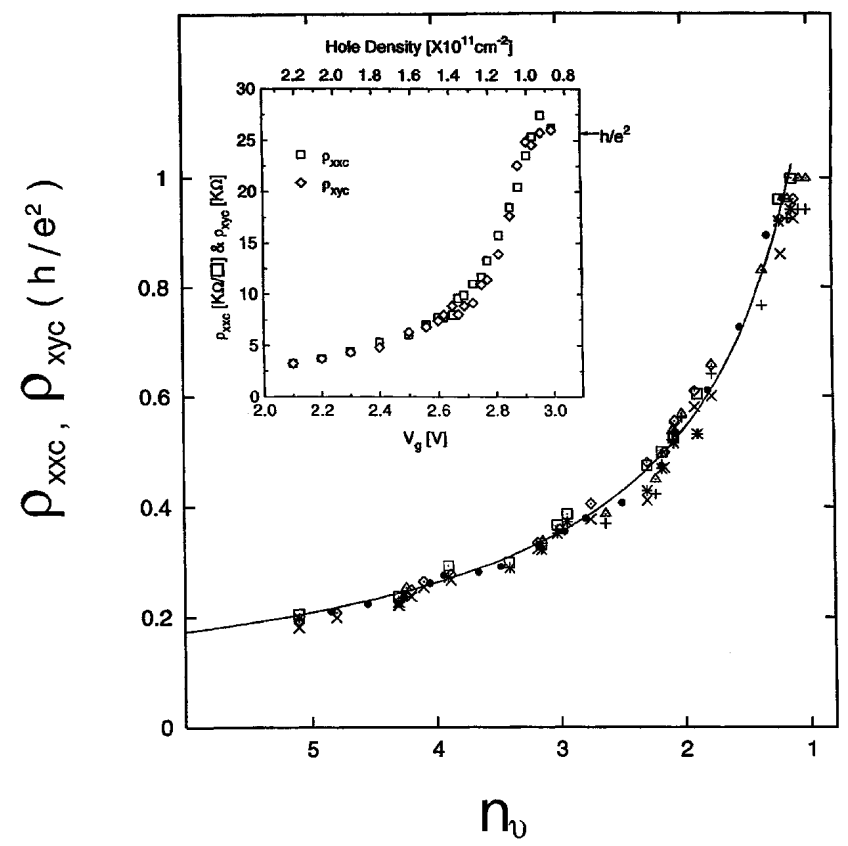

FIG. 2. Critical resistivities, $\rho_{x x c}$ and $\rho_{x y c}$, as function of Landau-level filling number $n_{\nu}$, respectively, at flux strength $\frac{2 \pi}{16}(\triangle,+) ; \frac{2 \pi}{24}(\diamond, \times) ; \frac{2 \pi}{32}(\square, *)$, and $\frac{2 \pi}{96}(\bullet$, none $)$. Inset: The experimental data by Song et al. [4].

side in the TBM. It is quite different from the well-known plateau-plateau transition $(\nu \rightarrow \nu \pm 1)$ within the IQHE regime as well as the transition from the IQHE state to insulator on the low-filling-number side (i.e., $\nu=1 \rightarrow$ $0)$ where, at the $\nu \rightarrow \nu+1$ transition, one has $\sigma_{x x c} \simeq$ $0.5 e^{2} / h$, while $\sigma_{x y c}=(0.5+\nu) e^{2} / h$ [10]. It provides a unique explanation for recent experiments $[3,4]$. In order to further understand this new universality of critical behavior at the high-filling boundary of the IQHE, we investigate the scaling behavior in this regime below.

According to the general scaling theory of the QHE system [14], the finite-size localization length may be written as a general function of two parameters, i.e., $\lambda_{L} / L=f(L / \xi, p)$, at a large sample with a width $L$. Here the first parameter is $L$ divided by the thermodynamic localization length $\xi$ as usual [13], and the second one, $p$, may be chosen as either $\sigma_{x x}$ or $\sigma_{x y}$ at a fixed sample size $L_{0}$. In the present case, it is convenient to choose $\sigma_{x x c}$ as $p$ (which can be connected with $\sigma_{x x}$ and $\sigma_{x y}$ at $L=L_{0}$ by the scaling-flow diagram [14]). Based on this scaling hypothesis, the numerical data at different flux strength $2 \pi / M$ 's, disorder strength $W$ 's, sample size $L$ 's, etc., should all collapse into a single curve so long as the critical conductance $\sigma_{x x c}$ is the same. Indeed, as shown in Fig. 3, the data of finite-size localization lengths can be well fit into one curve by choosing a single scaling variable $L / \xi$ at the given $\sigma_{x x c}=1.06$. Such a $\sigma_{x x c}$ corresponds to a $\nu=2 \rightarrow 0$ transition. By changing the plateau index $\nu$ or the Fermi energy $E_{f}$, the critical conductance $\sigma_{x x c}$ can change continuously as discussed before. One expects the

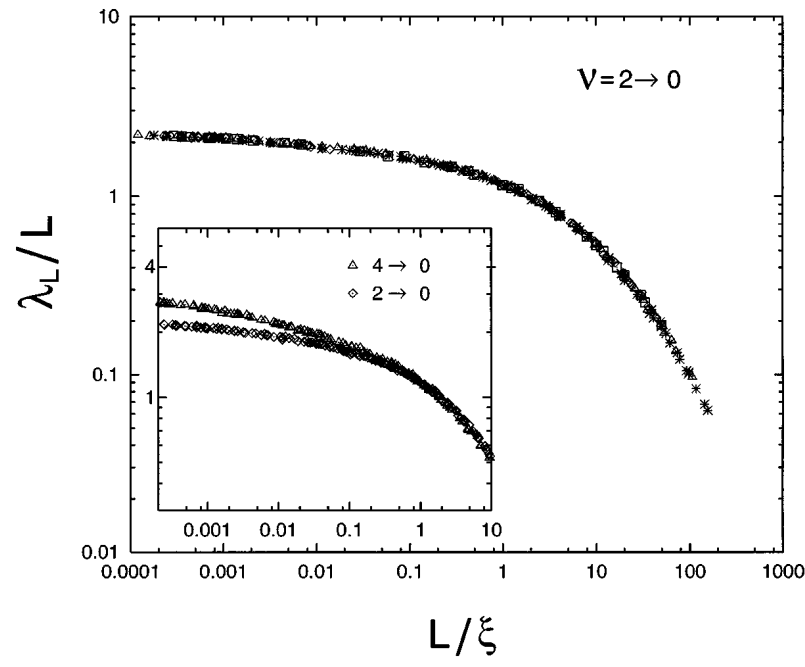

FIG. 3. Two-parameter scaling function $\lambda_{L}\left(L / \xi, \sigma_{x x c}\right) / L$ versus $L / \xi$ at $\sigma_{x x c}=1.06$. All the data at different disorders collapse into one curve with the stripe sample width $L$ varying from $L=16$ to 80 and flux strengths from $\frac{2 \pi}{16}(*), \frac{2 \pi}{24}(\diamond)$, $\frac{2 \pi}{32}(\square)$ to $\frac{2 \pi}{96}(\triangle)$. Inset: Two different scaling curves for the $\nu=2 \rightarrow 0\left(\right.$ at $\left.\sigma_{x x 2}=1.06\right)$ and $\nu=4 \rightarrow 0\left(\right.$ at $\left.\sigma_{x x 4}=2.10\right)$ transitions, respectively.

scaling curve to change correspondingly. In the inset in Fig. 3, scaling curves at two critical points $(\nu=2 \rightarrow 0$ and $\nu=4 \rightarrow 0$ ) are shown with $\sigma_{x x 2}=1.06$ and $\sigma_{x x 4}=$ 2.1 , respectively. Different scaling curves have also been obtained for the transitions of $\nu=3 \rightarrow 0$ and $\nu=1 \rightarrow$ 0 in this regime. All of these different cases can be generally specified by a single LL filling number $n_{\nu}$, since $\sigma_{x x c}$ is uniquely determined by $n_{\nu}$ as shown in Fig. 2. Therefore, a two-parameter scaling law is well established here which characterizes a new universality of metal-insulator transitions between the IQHE regime and insulator at high-filling-number boundary. It is noted that the transition from the IQHE state to insulator at lowfilling-number boundary (corresponding to $\nu=1 \rightarrow 0$ ) is usually well described by a one-parameter scaling law [11], which may be understood as the second parameter $\sigma_{x x c}$ always remains a constant in this regime [10]. So the present case is the first one in the QHE systems that a two-parameter scaling law becomes necessary in order to describe the critical behavior. Finally, if one plots the finite-size conductance $\sigma_{x x L}$ as a function of the finitesize localization length $\lambda_{L} / L$ at different flux strengths $(2 \pi / 16-2 \pi / 96)$, as well as different plateau index $\nu$ 's, the Fermi energy $E_{f}$ 's and disorder strength $W$ 's, all the data also collapse into one curve as shown in Fig. 4. (The notations of the data in Fig. 4 are similar to those in Fig. 3.) It means that $\sigma_{x x L}$ is uniquely decided by $\lambda_{L} / L$, and thus also satisfies the two-parameter scaling law.

Finally, we point out that in the above discussion, we have not considered the spin degrees of freedom. For the conventional plateau-plateau transition, it is well known [15] that the mixing of LL's for different spins by a 


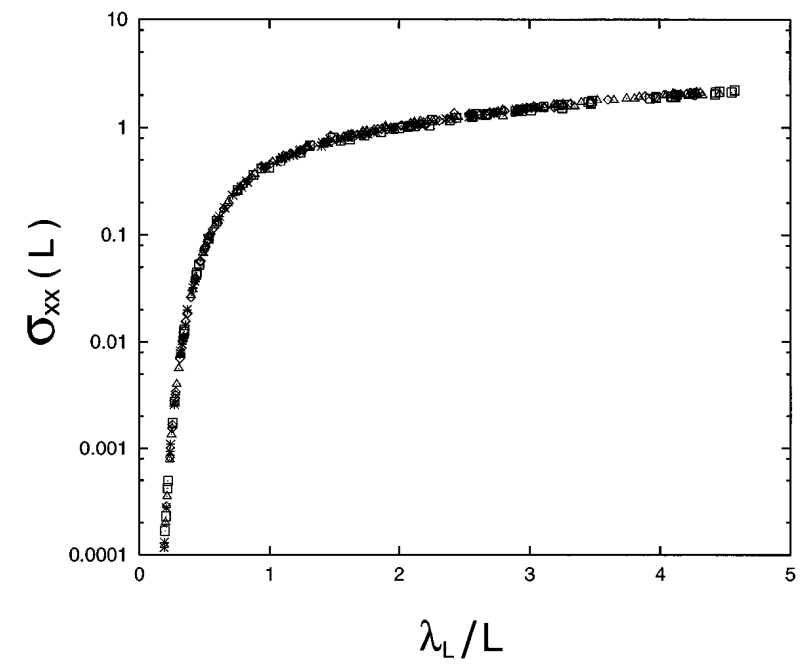

FIG. 4. Finite-size conductance $\sigma_{x x L}$ as a single function of $\lambda_{L} / L$ at different sample sizes, flux strengths, plateaus indexes, disorder strengths, and Fermi energies.

spin-orbit coupling will not change the universality class and such a spin-coupled system behaves still like a spinless system. In our case, we have checked that introducing a weak spin-orbit coupling effect will also make the system with spin degrees of freedom behave like a spinless system. For example, the $\nu=2 \rightarrow 0$ and $\nu=3 \rightarrow 0$ transitions remain to be direct transitions (even with Zeeman splitting) with the same universality of critical behavior discussed above for the spinless case. Both diagonal and Hall conductance are also equal to each other and fall into the same range shown in Fig. 2.

In summary, we have identified a new two-parameter scaling law in the critical regime of direct transitions from the high IQHE states to insulator in the TBM. Such a new universality of metal-insulator transitions predicts that $\rho_{x x c}=\rho_{x y c}$ and their value depends solely on the LL filling number $n_{\nu}$. The critical behavior of this lattice model in the aforementioned direct phase transition regime provides a consistent explanation for recent experimental measurements.
The authors thank T. Xiang, D. Shahar, S. V. Kravchenko, K. Yang, and C.S. Ting for stimulating and helpful discussions. The present work was supported by Texas Center for Superconductivity at University of Houston, and grants from Robert Welch foundation and Texas Advanced Research Program.

[1] S. Kivelson, D. H. Lee, and S. C. Zhang, Phys. Rev. B 46, 2223 (1992).

[2] H. W. Jiang et al., Phys. Rev. Lett. 71, 1439 (1993); T. Wang et al., Phys. Rev. Lett. 72, 709 (1994).

[3] S. V. Kravchenko et al., Phys. Rev. Lett. 75, 910 (1995); D. Shahar et al., Phys. Rev. B 52, R14372 (1995), and references therein.

[4] S.-H. Song et al., Phys. Rev. Lett. 78, 2200 (1997).

[5] M. M. Fogler and B. I. Shklovskii, Phys. Rev. B 52, 17366 (1995); A. M. Tikofsky and S. A. Kivelson, Phys. Rev. B 53, 13275 (1996).

[6] D. E. Khmel'nitzkii, Phys. Lett. 106A, 182 (1984).

[7] R. B. Laughlin, Phys. Rev. Lett. 52, 2304 (1984).

[8] D. Z. Liu, X. C. Xie, and Q. Niu, Phys. Rev. Lett. 76, 975 (1996); Phys. Rev. B 54, 4966 (1996).

[9] D. N. Sheng and Z. Y. Weng, Phys. Rev. Lett. 78, 318 (1997).

[10] S. A. Kivelson, D.-H. Lee, and S.-C. Zhang, Phys. Rev. B 46, 2223 (1992); Y. Huo, R. E. Hetzel, and R. N. Bhatt, Phys. Rev. Lett. 70, 481 (1993); Z. Wang, B. Jovanovic, and D.-H. Lee, Phys. Rev. Lett. 77, 4426 (1996).

[11] B. Huckestein and B. Kramer, Phys. Rev. Lett. 64, 1437 (1990), and references therein.

[12] D. S. Fisher and P. A. Lee, Phys. Rev. B 23, 6851 (1981); H. U. Baranger and A. D. Stone, Phys. Rev. B 40, 8169 (1989).

[13] A. MacKinnon and B. Kramer, Phys. Rev. Lett. 47, 1546 (1981); Z. Phys. B 53, 1 (1983).

[14] A. M. M. Pruisken, Phys. Rev. Lett. 61, 129 (1988); H. Levine, S. B. Libby, and A. M. M. Pruisken, Phys. Rev. Lett. 51, 1915 (1983).

[15] D. Lee and J. Chalker, Phys. Rev. Lett. 72, 1510 (1994); Z. Q. Wang, D.-H. Lee, and X. G. Wen, Phys. Rev. Lett. 72, 2454 (1994). 